\title{
The new year for chromosome research: a change of guard amidst a shifting scientific landscape and global pandemic
}

\author{
Beth A. Sullivan
}

Accepted: 13 January 2021 / Published online: 24 February 2021

(C) The Author(s), under exclusive licence to Springer Nature B.V. part of Springer Nature 2021

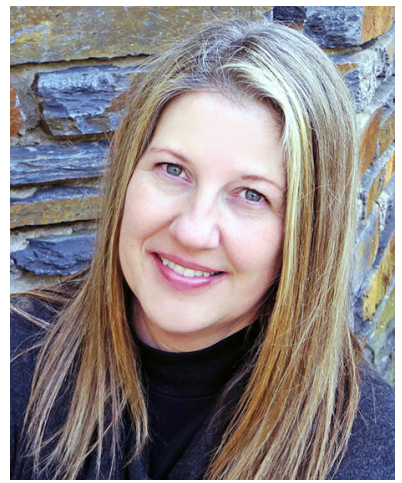

Beth Sullivan, $\mathrm{PhD}$, new Editor-in-Chief of Chromosome Research

On December 31, 2020, after 10 years as Editor-inChief, Dr. Conly L. Rieder stepped down from the journal and officially retired. I assumed the role of the next Editor-in-Chief (EiC) of Chromosome Research on January 1, 2021. I look forward to the exciting experience of leading the journal and working with the Springer Nature editors and the talented and engaged researchers on the editorial board.

The late Professor Herbert MacGregor co-founded the journal Chromosome Research in 2003 with Drs. Pat Heslop-Harrison and Michael Schmid. Herbert, a chromosome biologist who was renowned for his seminal research

Responsible Editor: Stefanie Dether

B. A. Sullivan $(\square)$

Department of Molecular Genetics and Microbiology, Division of Human Genetics, Duke University School of Medicine, DUMC 3054, Durham, NC 27710, USA

e-mail: beth.sullivan@duke.edu on lampbrush chromosomes (Gall 2018), launched Chromosome Research as a chromosome-focused journal that aimed to be affordable, published high-quality research, and had an efficient editorial process. He worked tirelessly to publish chromosome studies from many different organisms and invited a broad mix of chromosome researchers, including Conly and myself, to join the Editorial Board to support the journal's scientific mission. In 2010, Herbert started training Conly to succeed him as EiC, and for 18 months, they worked together to steer the journal as Conly learned the ropes of scientific publishing.

Conly was a natural successor to Herbert due to his long-standing research expertise in mitosis and chromosome movement and his deep love and larger-than-life enthusiasm for chromosome biology. Many of us still recall Conly's talks at scientific conferences in which he showed live cell movies of a PtK1 chromosome that had been laser-severed from the mitotic spindle. Its reengagement with the microtubules and forceful pull toward the spindle midzone would be punctuated by Conly's loud hand clap and a "BAM!" delivered in his booming voice. Conly spent decades studying the mechanics and molecular behaviors of vertebrate chromosomes as they moved through the cell cycle. His contributions spanned spindle-kinetochore interactions, centrosome assembly, mitotic chromosome behavior, spindle dynamics, and mitotic checkpoints, all captured in his beautiful time-lapse movies of newt lung cell mitoses or cell fusions of rat kangaroo (PtK1) cells and stunning high-resolution fluorescence or electron microscopy images of centrosomes and mitotic chromosomes attached to spindle microtubules. 
During his tenure as EiC of Chromosome Research, Conly and his Associate Editors (AEs) surveyed the scientific landscape in the field, updating the Aims and Scope of the journal to welcome findings on chromatin, chromosomes, and genomes in a variety of organisms. Conly and the AEs refocused the publishing priority of the journal to report scientific discoveries that provided new functional knowledge of chromosomes and genomes, moving beyond important but largely observational findings that had been a hallmark of chromosome studies for several decades. This new philosophy resulted in a more stringent acceptance rate, from $38 \%$ in 2012 to a steady average acceptance rate of $24 \%$ (2014-2019). Conly and his AEs also pushed hard to provide authors with better benefits and experiences, such as a quicker time to first decision (16 days in 2013 to 11 days in 2020) and faster submission to online publication ( $<60$ days in 2019-2020). While journal impact factor (IF) is not the only metric by which a journal's performance is measured, it still remains an element of how a journal is perceived by readers and authors alike. Under Conly's leadership, Chromosome Research's IF rose from 2.478 in 2014 to 3.413 in 2019, attesting to the scientific influence of the papers that were published and cited, particularly in recent years.

Historically, 1-2 issues of the four annual Chromosome Research issues have been designated as Special Issues (SIs). These thematic issues, guest-edited by leading experts in a specific topic in chromosome biology, typically contain 6-14 review or original research articles authored by prominent scientists. The focused nature of the SIs gives readers a broad view on a specific topic that provides historical context but primarily highlights contemporary findings and key areas of future exploration. The SI articles are among Chromosome Research's most read and cited material and have focused on exciting topics such as Ribosomal DNA (2019), Transposable Elements and Genome Function (2018), Genome Organization (2017), and Repetitive DNA (2015). Readers should look forward to the 2021 Special Issue on "Imaging Approaches to Chromosome Structure", guest edited by Professor Kiichi Fukui (Osaka, Japan) and Dr. Toshiyuki Wako (Tsukuba, Japan).

The Special Issues are where I "cut my teeth" as an editor at Chromosome Research. After being asked by Herbert MacGregor to join the Associate Editor Board in 2010, I co-guest edited a Special Issue on Centromeres in 2012 with Dr. Rachel O'Neill (University of Connecticut, Storrs, USA). Due partly to this positive experience with the EiC, journal staff, and the authors as well as to the success of that particular Special Issue, Conly appointed me in 2013 as the Executive Editor with responsibility for annual Special Issues. From 2013 to 2020 , I planned and provided oversight for 10 Special Issues. I was fortunate to work with many different SI guest editors-outstanding researchers from different subfields of chromosome and nucleus biology. During this period, I also learned the journal trade from Conly, as he included me in many editorial decisions. Our level of engagement, especially over the past year in which we served together as co-EiCs, coupled with Conly's drive and focus to elevate the journal's reach and influence, unquestionably prepared me to take on the leadership of the journal in 2021 as Conly steps down and moves into well-deserved retirement.

\section{What's next for chromosome research?}

A change of leadership provides an opportunity to reassess the landscape of chromosome biology, scientific publishing, and research training in order to modify where needed while continuing to elevate the journal's mission. Herbert, Conly, and the journal publishers (now Springer Nature) have established a solid infrastructure for receiving, handling, and publishing submissions that have reflected the past 30 years of chromosome biology. From the first papers published in 1993 that described the segregation of holocentric chromosomes in C. elegans (Albertson and Thomson 1993) and scanning electron microscopy of synaptonemal complexes in plants and mammals (Barlow et al. 1993) to our more recent publications reporting on cell cycle-mediated DNA methylation changes (KubiuraIchimaru et al. 2020) and oligonucleotide probes to universally identify chromosomes from different Zea species (Braz et al. 2020), the journal has spanned an incredible breadth of chromosome-related research. Supported by innovative and diverse technologies and approaches, chromosome biology and genome research has evolved over the past 30 years.

\section{Scope}

Going forward, the focus of the journal will, of course, remain on chromosome biology, including chromatin biology, epigenetics, genome organization, and 
structure/function of the nucleus. We will continue to publish studies that address foundational, hypothesisbased questions of topics in chromosome and genome organization and function. However, chromosome biology has changed dramatically since the inception of the journal, as technologies have developed and matured. Now, chromosome biology includes new advanced microscopy and fluorescence instrumentation, customized probe sets for high resolution, next-generation molecular cytogenetics, nanotechnology to improve fluorescence-based assays, inducible tagged protein tracking and degradation, precise chromosome and genome engineering, and high-throughput global (epi)genomic approaches. Chromosome Research welcomes new submissions that incorporate these modern cytogenetic, molecular, and genomic techniques, alone or in parallel with established stalwart approaches. The Associate Editors and I plan to update the journal's Aims and Scope to reflect our commitment to publishing papers that employ the latest technologies and approaches to address fundamental questions in chromosome and genome biology.

\section{Content}

Chromosome Research has historically published original research and review articles. Most reviews have been published as part of thematic Special Issues. We expect to continue publishing an annual Special Issue, and I am happy to announce that as I have moved to the role of EiC, Dr. Rachel O’Neill (University of Connecticut, Storrs) has agreed to serve as Executive Editor in charge of Special Issues. Rachel is a quintessential chromosome biologist-her research spans molecular cytogenetics, centromere function, chromosome and genome stability and evolution, small RNA biology, retroelement and transposon biology, repetitive DNA biology, and genetics and genomics in eukaryotes. She has been a frequent contributor to Chromosome Research and has served as an Associate Editor for several years. Rachel is also a leader in genomic technologies and will be a key catalyst, along with several of our current and new Associate Editors, in ensuring that our future publications reflect the breadth of next-generation technologies used in chromosome and genome research. I am thrilled to be working with Rachel in this new capacity, and with the advice of our outstanding Associate Editors and Editorial Advisory Board, we plan to showcase exciting topics in our future Special Issues.
Chromosome Research will continue to publish a limited number of reviews that cover the historical to contemporary context of a specific chromosome biology-related topic as part of the WaldeyerFlemming Special Collection. Readers will also find the occasional review or mini-published with the original research articles in our 3 regular issues. Authors are encouraged to email a pre-submission inquiry to any Associate Editor or me and we will gauge the suitability of a topic or submission for the Waldeyer-Flemming Special Collection, a standalone review, or an opinion piece.

We are also excited to launch a few new features of the journal. "Method/Protocol/Resource" will be a new article type by which authors can share information on new tools, protocols, and experimental or computational approaches that are broadly applicable and of potentially of high interest to the community. Other new features will include interview-style articles that profile members of our Editorial Board and their research. We want readers and contributors to become familiar with our editorial board so they can identify one or more suitable editors who could shepherd their future submissions through the review process. The journal will also publish short articles that highlight noteworthy established and emerging scientists in chromosome biology, epigenetics, genome organization and function, and chromatin biology. The new editor and research profiles will aim to create a stronger community, by building awareness of the exciting and diverse science happening across the globe and highlighting the creative individuals driving the research. I encourage you to visit the journal web page often for more information and an opportunity to suggest early, mid-, or late career researchers to be profiled.

Publishing options - moving to becoming fully open access

One of Herbert MacGregor's original visions for Chromosome Research was for it to be fully open access. Currently Chromosome Research is a hybrid journal. When research is accepted for publication, authors choose to publish using either the traditional publishing route or gold Open Access (OA), available at Chromosome Research as "Open Choice." However, Chromosome Research is actively committed to becoming a fully OA 
journal, and as EiC, I fully support this move to publishing research that is affordable and accessible to all. We aspire to increase the number of OA articles over the next year or two, with the goal of becoming fully OA. A journal that commits to this process is known as a Transformative Journal (TJ); this is Chromosome Research's current designation. Publishing via the gold OA route in Chromosome Research is compliant with PlanS OA requirements, and cOAlition $\mathrm{S}$ funders, such as Wellcome Trust, Howard Hughes Medical Institute, and other national research funders, can support article processing charges (APCs). To learn more about Chromosome Research's pledge to becoming fully OA and how you can join us in our mission, visit https://www. springer.com/journal/10577/open-access-publishing.

\section{Diversity and equity}

Being a female researcher and administrator at my university, I am aware of the disparities and lack of diversity in the biomedical sciences and in the leadership of many biomedical journals. As I take the helm of the journal in 2021 as its first female Editor-in-Chief, I intend to engage established scientists and junior scientists/trainees alike as we look to the future of science communication and specifically Chromosome Research's place as a scientific publication among many other biomedical science journals. One goal that is shared by many on our Editorial Board is to increase diversity among the editors - in geographical location, gender, ethnicity, scientific expertise, and career stage. We also hope to engage more with trainees to give them the opportunity to understand and learn about the inner workings of the editorial process. Having a variety of input and feedback on the journal's course and activities will lend to wise editorial decisions that will ensure the journal continues to provide content that matches our readers' needs and preferences and that will sustain and grow the readership through the next generations of chromosome biologists.

So as I write this editorial on the last day of December 2020, I offer my heartfelt thanks to Dr. Conly Rieder for his vision and commitment to the journal over the past 10 years and for his support and guidance as I prepared for the role of the next Editor-in-Chief. I wish Conly the best of everything in his retirement, especially as he resumes his travels to exciting, far-flung destinations around the world...well, once current travel restrictions are relaxed, that is! This past year has been extremely challenging, dominated by a global pandemic that made many feel disconnected, less productive, and vulnerable. I look forward to serving the scientific community and building new and deeper connections with authors, reviewers, fellow editors, trainees, and colleagues. As we enter 2021, I wish you all joy, peace, hope, health, and many successful chromosome-based experiments!

\section{References}

Albertson DG, Thomson JN (1993) Segregation of holocentric chromosomes in the nematode, Caenorhabditis elegans. Chromosome Res 1:15-26

Barlow AL, Jenkins, ap Gwynn I (1993) Scanning electron microscopy of synaptonemal complexes. Chromosome Res 1: 9-13

Braz GT, do Vale Martins L, Zhang T, Albert PS, Birchler JA, Jiang J (2020) A universal chromosome identification system for maize and wild Zea species. Chromosome Res. 28:183194

Gall JG (2018) Herbert MacGregor (1933-2018). Chromosome Res. 26:225-331

Publisher's note Springer Nature remains neutral with regard to jurisdictional claims in published maps and institutional affiliations. 